\title{
Wide Range Fire Extinguish System for Residential Use
}

\author{
Kouki Yamaguchi ${ }^{\mathrm{a}}$, Hayato Takahashi ${ }^{\mathrm{a}}$, Masahiko Hanada ${ }^{\mathrm{b}}$, Hiromichi Hanada ${ }^{\mathrm{b}}$, Kanya Mizuguchi ${ }^{\mathrm{b}}$, \\ Akinori Fujimoto $^{c}$, Seiichi Serikawa ${ }^{\mathrm{d}}$, Yuhki Kitazono*
}

${ }^{a}$ National Institute of Technology, Kitakyushu College, 5-20-1 Shii, Kokuraminami-ku, Kitakyushu-city Fukuoka 802-0985, Japan

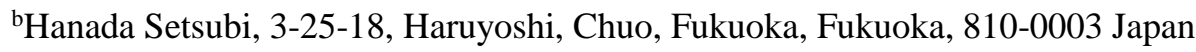

${ }^{\mathrm{c}}$ Fukuoka City Council, 1-8-1, Tenjin, Chuo, Fukuoka, Fukuoka, 810-8620 Japan

${ }^{d}$ Kyushu Institute of Technology, 1-1, Sensui-cho, Tobata, Kitakyushu, Fukuoka, 804-8550 Japan

*Corresponding Author: kitazono@kct.ac.jp

\begin{abstract}
We has improved a system that detects a fire and performs initial extinguishing. The features of the system are installable by attaching to extinguisher and controllable through web browser. In addition to these, the system detects a fire automatically by processing the image from infrared camera with OpenCV, and send alert to user. We developed the system in the previous research, and this research we improved the system to enable it to extinguish a fire in a wide range and to make it easier to install to indoor place.
\end{abstract}

Keywords: fire extinguishing, OpenCV, Raspberry Pi.

\section{Introduction}

Total number of fires occurred in Japan in 2013 is 48,095 and total number of the deceased is $1,625^{(1)}$. Building fire accounts for 25,053 of total number of fires. Besides, the economic damage per fire amounts to 12 million yen. The most effective way to prevent such damage by fire is initial extinguishing. Initial extinguishing is extinguishing a fire in 2 minutes from time the fire has occurred. By extinguishing fire before it spreads, damage is drastically reduced. In addition, the activity of fire engine is also reduced. Therefore, initial extinguishing is an efficient way of fire extinguish. There is great difference in damage between the cases initial extinguishing is performed and the other cases.

However, initial extinguishing is not performed in many cases contrary to its importance. It is conceivable that man is not aware of the fire as a reason why initial extinguish is not performed. The situation man was asleep when the fire occurred or that man is not at home. Performing initial extinguishing in such situation is so difficult. The fire becomes bigger silent in consequence. Besides, it is also difficult to extinguish a fire occurred in housing for elderly because the elder may not be able to extinguish the fire properly due to his old body. Thereby, dead incidence of people over 65 years old is ten times higher than people younger than 65 years old.

The systems for performing extinguishing is already exist. For example, home fire alarm is low in price and installable in any house. However, it doesn't have an extinguishing function. Additionally, when it worked and alarm was raised, fire may become so big that it is not at initial extinguish stage. For more examples, sprinkler has high fire extinguishing ability, but it sprinkles a large amount of water all over the area. Owing to its way to extinguish, the area is soaked with water even it succeeded in extinguishing fire, hence electronic equipment like computers can break down. To take advantage of initial extinguish, we need a system that detects fire in initial stage and extinguishes the detected fire properly.

From such a background, we had built a system that performs initial extinguishing by remote control last time ${ }^{(2)}$. However, the system is intended to detect fire by setting an IR camera near one of the fire sources. Therefore, it was problem that appropriate detection for multiple fire sources was difficult. Therefore, we improved the system so that it can detect fire properly for multiple fire sources.

As another improvement the system has a range of movement in horizontal axis. So the accuracy at extinguishing has increased. 


\section{Structure}

\subsection{Structure of body}

The system is set in home and intended to perform fixed point extinguishing. It is consisted of fire extinguisher, video camera, infrared camera and Raspberry Pi. The core of the system is Raspberry Pi. Raspberry $\mathrm{Pi}$ is about business card size computer. Linux runs on Raspberry Pi and IO-pins are on the body. In short we can control the system with Linux OS and control devices such as motor and other electric devices with IO-pins.

The operation schematic diagram of the system is shown in Fig. 1. First, if fire occurs, fire detection system detects the breaking out of fire. Then, a notification is sent to user and user accesses the website for fire extinguishing via device user owns such as smartphone, tablet or any device which has web browser. If user makes sure that fire has actually occurred and presses the extinguishing button on the website, the system makes the fire extinguisher work.

Infrared images are sent to Raspberry Pi from infrared camera, and these are processed with image processing program. If the system has detected a fire, user confirms that the fire has actually occurred with video images from camera. And then the system performs extinguish.

\subsection{Structure of System}

The system is divided into two major units; fire detection unit that monitors whether a fire has occurred, and extinguish unit that extinguishes a fire. The schematic diagram of the system is shown in Fig. 2

Fire detection unit is consisted of infrared camera and Raspberry Pi. Infrared camera catches only infrared light. Because flare emits infrared, the system can detect size of flare by watching the shape of the infrared. If the system detected a fire, send email to preset email address. Then, user accesses the website for controlling the system from URL on the email.

Extinguish unit is consisted of video camera, Raspberry Pi, and fire extinguisher. There are two parts on the website user accesses to control the system; a real time video image and fire extinguishing button. User confirms that the fire has occurred actually with video camera image, and then performs extinguishing by pushing the extinguishing button.

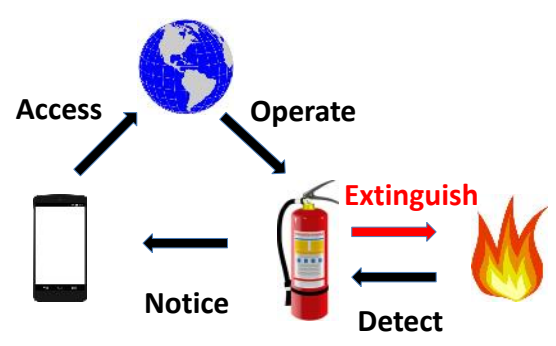

Fig. 1. Operation schematic diagram.

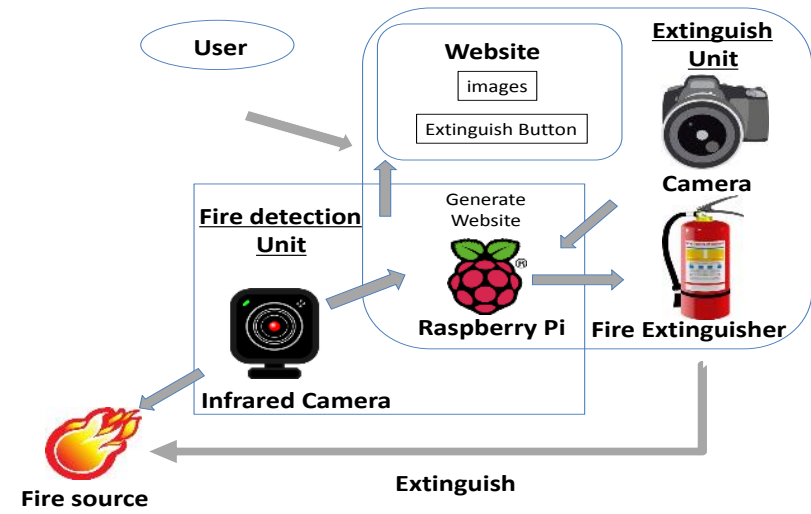

Fig. 2. Schematic diagram of the system.

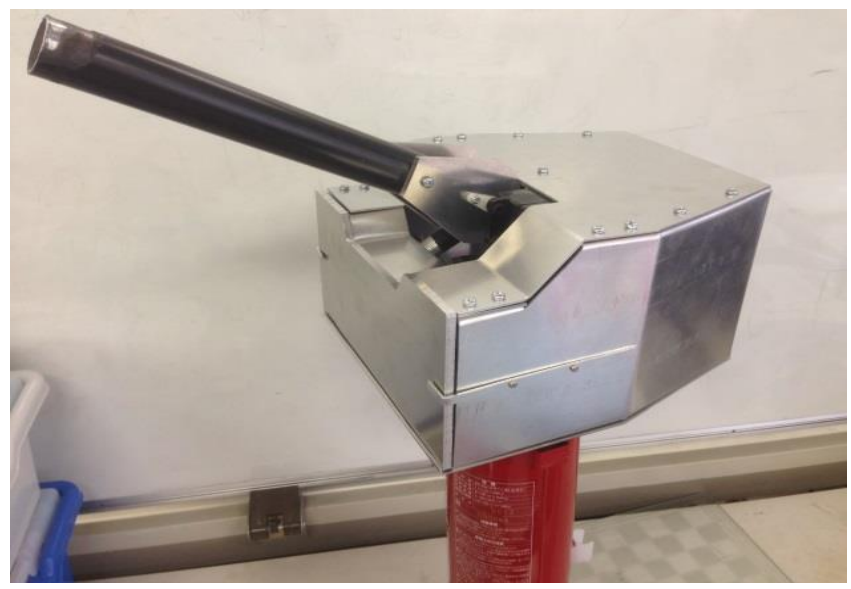

Fig. 3. Previous research exterior ${ }^{(3)}$.

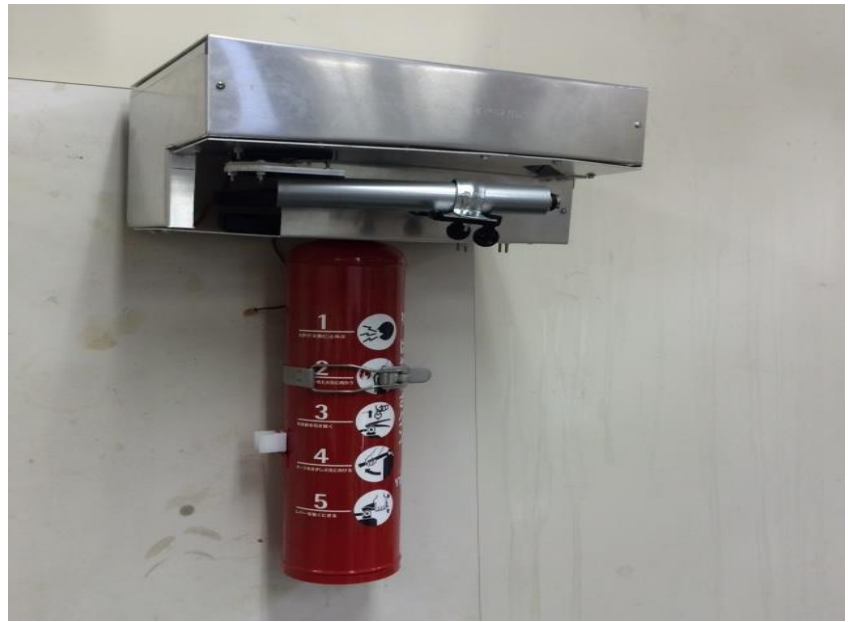

Fig. 4. Improved exterior. 


\subsection{Exterior Design}

A main system has improved the exterior of the system which previous research exterior ${ }^{(2)}$. Improvement points is listed below. First, the system hooked on the wall. Second, it is that range of the range of motion of the fire extinguisher hose has changed from the vertical axis to the horizontal axis. As a result, the system can aim the fire point is wide range. Previous research exterior is shown in Fig. 3 and improved exterior is shown in Fig.4.

The hose of the fire extinguisher is covered through the aluminum pipe which is attached to servomotor. The servomotor is the mechanism that the hose which is fixed by the thing which revolves through an aluminum pipe moves to a transverse. A simple mobile figure of the hose part is shown in Fig.5. The main system will be installed in the four corners in the room like Fig.6 from a location part of an infrared camera on Fig.5.

In order to pull the Handle of the fire extinguisher, DC motor is installed at the side of Handle of the fire extinguisher. An iron wire is attached to the motor and wrapped around the handle of fire extinguisher. As the motor rotates, the wire is reeled up and pulls the handle of fire extinguisher. The actual manufactured exterior is shown in Fig. 7.

\section{System}

\subsection{Technology components of Fire Detection}

The fire is detected by processing the images from infrared camera with Raspberry Pi. OpenCV: open source image processing library is used for this fire detection system. The fire detection process is described below. First, Raspberry Pi gets the images from infrared camera. Fire source of the image is shown in Fig. 8 and image got it from the infrared camera is shown in Fig.9. To make it easier to detect the shape of fire, the camera image is converted into gray scale. Then, to remove noises, pixels with value under threshold are removed. Thereby, only the strong infrared is remained. Now, the image keeps only the shape of the fire. So the system can detect the size of the fire from the image. The system specifies the locations of fire sources set in advance. Specifically, the image from camera is divided into some compartments and user inputs the compartments that include the fire sources at startup of system. Images obtained by dividing the infrared image. Specifying the location of the fire sources previously, it is possible to monitor the place where a fire can occur. By monitoring the

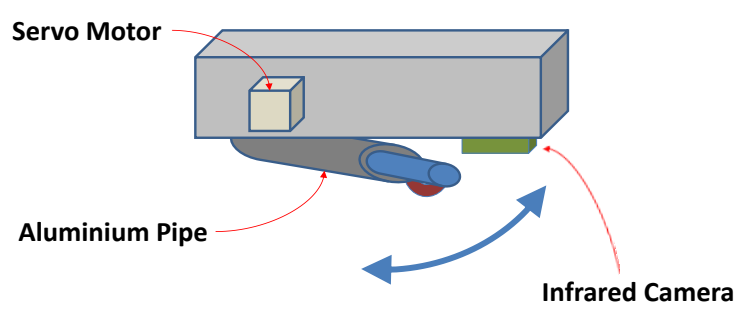

Fig. 5. Motion figure of the hose.

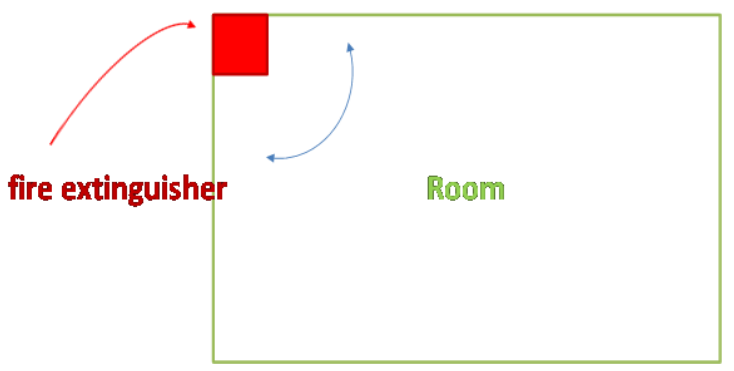

Fig. 6. Locating place.

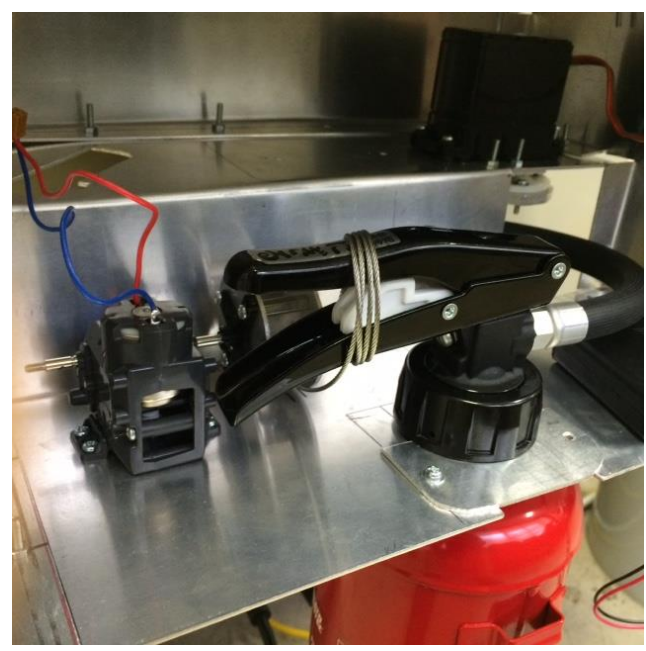

Fig. 7. Handle and DC motor.

fire sources intensively, breakout of fire can be found as soon as possible, so the detection accuracy is improved. The size of fire source is compared to the threshold that is set at system startup by recognizing normal size of the fire, if the size of fire source is greater than the threshold, it is recognized as fire. Image of after the image processing is shown in Fig.11. If the system detected the fire has occurred, it sends an alert mail to preset email address, and URL of website for firefighting is on the email. Then, user accesses the site from the URL, and performs extinguish through the site.

\subsection{Technology components of System}

The main component of the extinguish unit is website. User confirms that the fire has occurred and performs extinguish through the website. This system generates the 


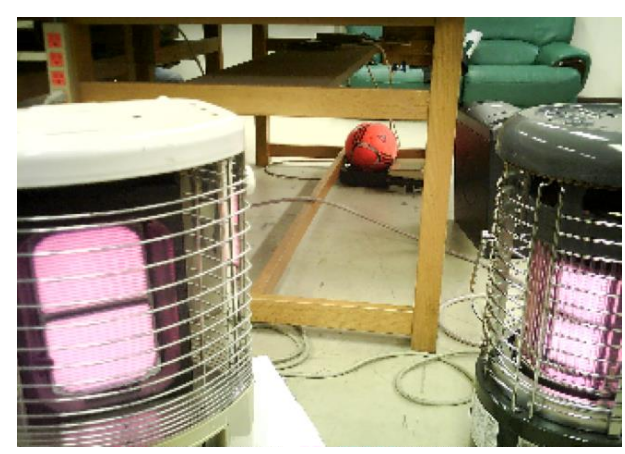

Fig. 8. Fire source of the image.

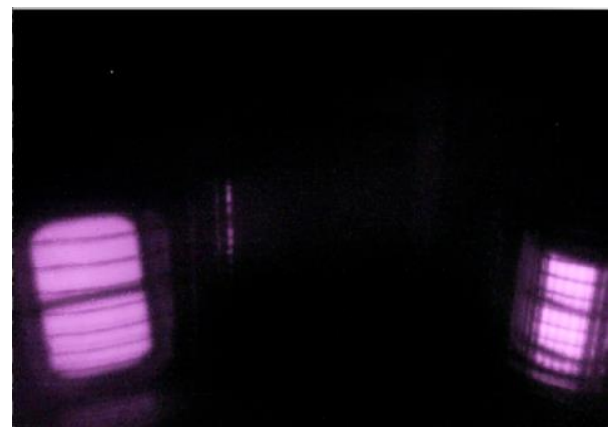

Fig. 9. Thermal imaging.

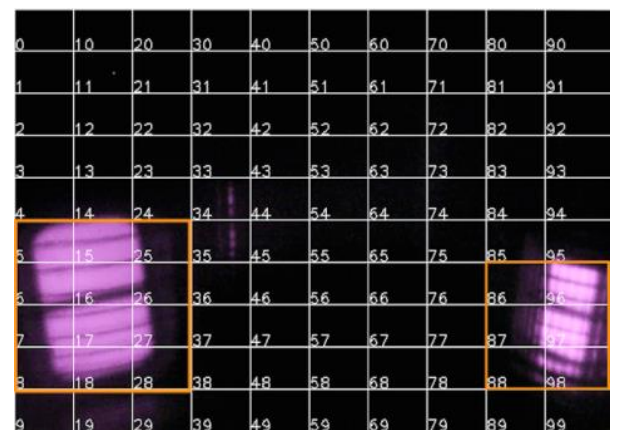

Fig. 10. Divided region image.

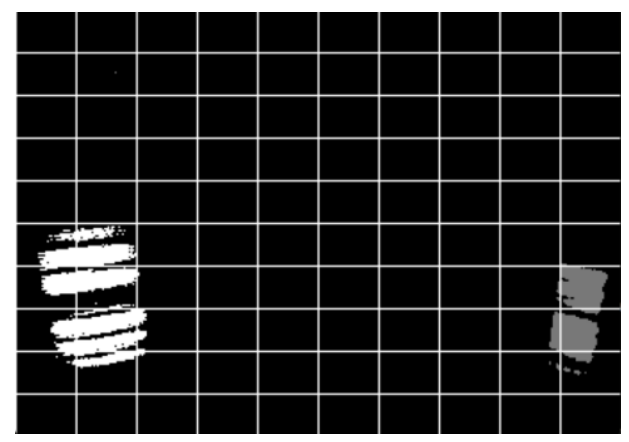

Fig. 11. Image of after the image processing.

website with WebIOPi: framework to generate website and enable Raspberry Pi to be controlled through the web browser. And this system gets the image used for confirming that the fire has occurred with MJPG-Streamer. MJPG-Streamer enables to Raspberry $\mathrm{Pi}$ to deliver the images from USB camera through HTTP. The website is generated by WebIOPi and shows the image from USB camera with MJPG-Streamer.

\section{Experimente}

The following two experiments were conducted to confirm the performance of the main system.

\subsection{Verification of fire detection}

As At first, we verified the fire detection whether it can detect a fire properly. Flame in sight of the camera. Then, the system would detect the fire. Therefore, we confirmed whether the system appropriate detect the fire.

\subsection{Verification of fire extinguish}

Secondly, we verified the fire extinguishing whether it can be operated by user's device and the system work properly. In this experiment, we access to the operation site which is generated by the system, and push the system is supposed to perform extinguish. By the procedure stated above, we confirmed the system can extinguish a fire.

\subsection{Verify whole the system operation}

Finally, we verify whole the system operation. The experimental procedure is described below.

(1) Locate the system on a wall.

(2) Generate a fire in sight of the camera.

(3) The system detect the fire and send mail to user's device.

(4) Access to the website with address on the mail.

(5) Operate the system with website and aim the fire.

(6) Push extinguish button and perform extinguish.

\section{Result}

\subsection{Result of fire detection experiment}

As a result, we confirmed that extinguishing system is detecting a fire source. The state of the image processing by the Raspberry pi is shown in Fig 12.

\subsection{Result of fire extinguish experiment}

As a results, we could extinguish with extinguishing system operation site. We confirmed that the motors has moved with each command of the fire extinguishing system operation site. Screen of the fire extinguishing system operation site is shown in Fig 13. 


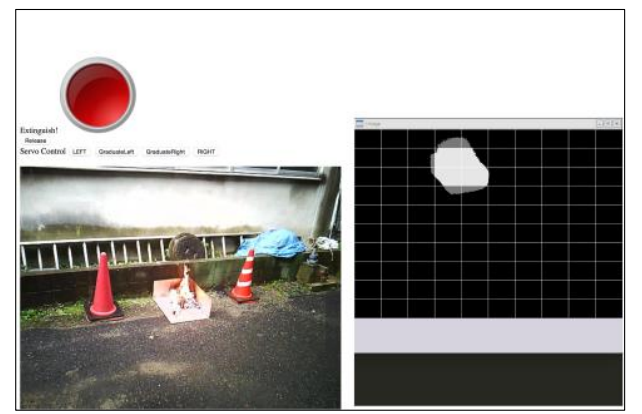

Fig. 12. The fire detection by image processing.

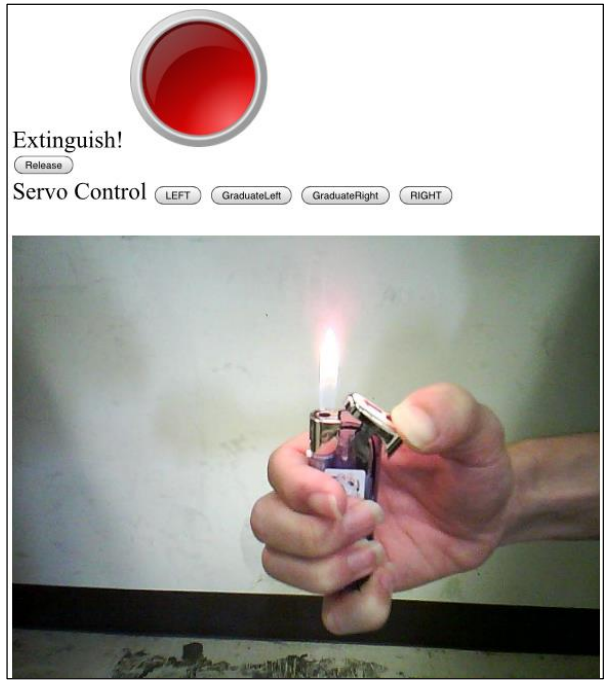

Fig. 13. Extinguishing command.

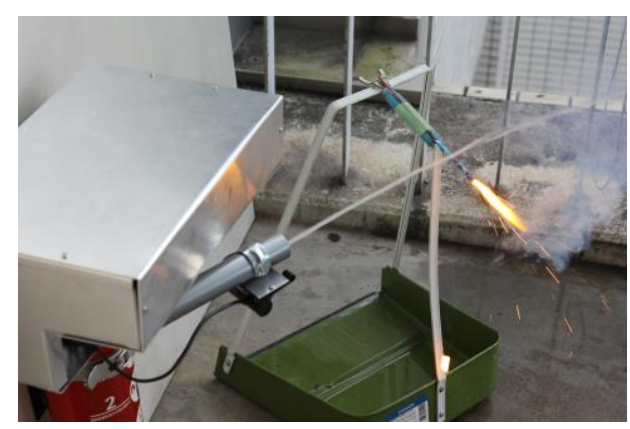

(a) Before extinguishing.

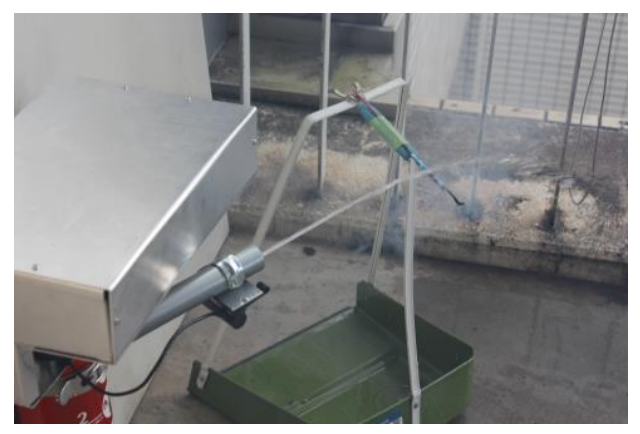

(b) After extinguishing.

Fig. 14. Simulated extinguishing experiment.

\section{$5.3 \quad$ Result of system operation}

As a result, we confirmed that the system of fire detection functioned tightly to fire extinguishing. The photos of before and after extinguishing by the system is shown in Fig. 14.

\section{Conclusion}

In As a result of experiment, extinguishing system in operation from the Web page is operating normally, it became more likely to extinguish indoor fire point by further moves on the horizontal axis. Future challenges, and more and more compact system, and it is to continue to so continue to spread world.

\section{References}

(1) Cabinet Office, Government of Japan : “Aged society white

paper".http://www8.cao.go.jp/kourei/whitepaper/w-201 2/zenbun/s1_2_1_03.html

(2) Hayato Takahashi, Masahiko Hanada, Hiromichi Hanada, Kanya Mizuguchi, Akinori Fujimoto, Seiichi Serikawa, Yuhki Kitazono, "Development of Automatic Fire Extinguish System for Residential Use", Proceedings of the 3rd International Conference on Industrial Application Engineering 2015, pp. 8-11, 2015 